\title{
A Decision-Theoretic Active Loop Closing Approach to Autonomous Robot Exploration and Mapping
}

\author{
Xiucai Ji, Hui Zhang, Dan Hai, and Zhiqiang Zheng \\ College of Mechatronics Engineering and Automation, \\ National University of Defense Technology, 410073 Changsha, China \\ \{jxc_nudt, zhanghui_nudt, haidan, zqzheng\}@ nudt.edu.cn
}

\begin{abstract}
One of the challenges of SLAM (Simultaneous Localization and Mapping) for autonomous robots is the loop closing problem. In this paper, a decision-theoretic active loop closing approach is presented, which integrates the exploration planning with loop closing. In our approach, the active loop closing process is modeled as a multi-stage decision problem, and a frontierbased auxiliary topological map is build to assist the decision process. The autonomous robot chooses its actions according to the sequential decision results. The unknown range most likely to close a loop is selected to explored, and a particle-filter-based localization and smoothing method applied to partial maps is used in the loop validating and loop constraints building process. Experiments have shown that our approach can practically implement loop closure and obviously improve the mapping precision compared to passive exploration strategy.
\end{abstract}

Keywords: SLAM, active loop closing, decision theory, particle filter.

\section{Introduction}

Simultaneous Localization and Mapping (SLAM) is the problem of learning maps of unknowns environment for a robot using the information obtained by sensors mounted on it, and it the key problem for the robot to be really autonomous [1]. A SLAM solution can be divided into at least the following three parts [2]:

- Incremental SLAM - The process of building an incremental map from sensor data, and simultaneously localizing itself.

- Loop Closing - The process of detecting if and where the robot entering an previously explored area.

- $\quad$ State Optimization - An optimization process that calibrates the map and robot localization (all the whole path) according to all the constraints from incremental mapping and loop closing.

It has been shown that SLAM is essential an incremental self-localization process, and the robot position estimation error will increase with the exploration of unknown area [3]. So it's difficult to detect loops by the incremental localization, and the loop closing problem is very critical to an autonomous robot to explore and map a large 
environment. If the robot hasn't the ability to close loops in its exploration, an area in the physical environment will correspond to multiple ones in its incremental map, and the map will be topologically inconsistent. On the contrary, if the robot has the loop closing ability, not only the consistent map will be obtained, but also the robot will know which area is truly unknown and explore the whole environment efficiently and reliably.

Some on-line loop closing approaches have been proposed [4-7], and they differ by the estimation algorithms (scan matching, Monte Carlo filter, etc.). Most of them are independent from the exploration strategy, and only when the robot occasionally meets a loop, the loop closing is activated, and some approach even assuming that the loop closing point (where the robot entering an previously explored area) is known. So these methods are passive.

In this paper, aiming at detecting and closing loops as soon as possible to reduce the mapping error, an active loop closing approach is presented, which combines the exploration strategy with the loop closing process. In our approach, the loop closing process is modeled as a multi-stage decision problem, and the robot selects its action according to sequential decisions. The robot actively explores the unknown areas more likely to close potential loops. When a potential loop is detected, a naval particle-filter-based globally robot localization algorithm in partial map is used in loop validation and then a path soothing approach is used to build multiple loop constraint. Experiments have shown that our approach can practically implement loop closure, and at the same time obviously improve the mapping precision compared to passive loop closing methods.

\section{Decision-Theoretic Architecture for Active Loop Closing}

Our active loop closing process is a combination of some sub-processes including exploration planning, loop detecting, validating and constraint building, and so on. We divide the exploration of the robot into two modes: regular exploration and loop closing exploration. In regular exploration mode, the robot explores the unknown area that most likely to close a potential loop, and at the same time it detects if it's possible that the robot has returned to a previously explored area, which is called loop detection. If it has, the robot enters the loop closing exploration mode to validate the potential loop. During the loop closing exploration, the robot needs to validate whether or not there is really a loop by a series of sensing perception, and this process is called loop validation. If the robot makes certain that there is a loop, it should then identify where the loop closure is, and create the loop constraints, and this process is constraint formation. If it's proven that there isn't a loop at the moment, then the robot returns to regular exploration.

During the loop closing process, the autonomous robot should make the following decisions to determine its actions:

\section{1) Loop Proposal Decision}

During the regular exploring, the loop detection should decide whether or not to start a loop closing exploration, and this is the loop proposal decision process, which tests the following tow mutually exclusive hypotheses: 
$H_{0}^{1}$ : There is a potential loop at the moment;
$H_{1}^{1}$ : There are no potential loops at all;

If $H_{0}^{1}$ is accepted, a new loop closing exploration is started. Otherwise, $H_{1}^{1}$ is accepted and the robot keeps the regular exploration.

2) Unknown Area Exploration Decision

During the regular exploration, there may be many unknown areas available for the robot to explore. In our approach, the unknown area exploration decision needs determine which one to explore allowing for the exploration cost and utility of every area.

3) Loop Validation Decision

It's possible that the loop detect process puts forward a spurious loop proposal, so every loop proposal must be checked carefully. The loop validation decision should test the following two tow hypotheses:

$$
\begin{aligned}
& H_{0}^{2}: \text { There is a loop at the moment; } \\
& H_{1}^{2} \text { : There isn't a loop at all; }
\end{aligned}
$$

It must be very carefully to make this decision, for if it's wrong, the robot will get an inconsistent map. If the robot couldn't determine anyone of the above hypotheses, it maintains the loop exploration for more sensing evidences.

Fig. 1 shows the basic scheme of the active loop closing process. The active loop closing process is driven by the results of sequential decision processes. At the beginning of every time step of the regular exploration, the loop proposal decision process determines whether or not to propose a potential loop closure. If $H_{1}^{1}$ is accepted, after loop closing constraints are constructed, the regular exploration will continue, and the unknown area exploration decision process will select the best area to explore. If $H_{0}^{1}$ is accepted, the loop closing exploration will be activated, and the loop validation

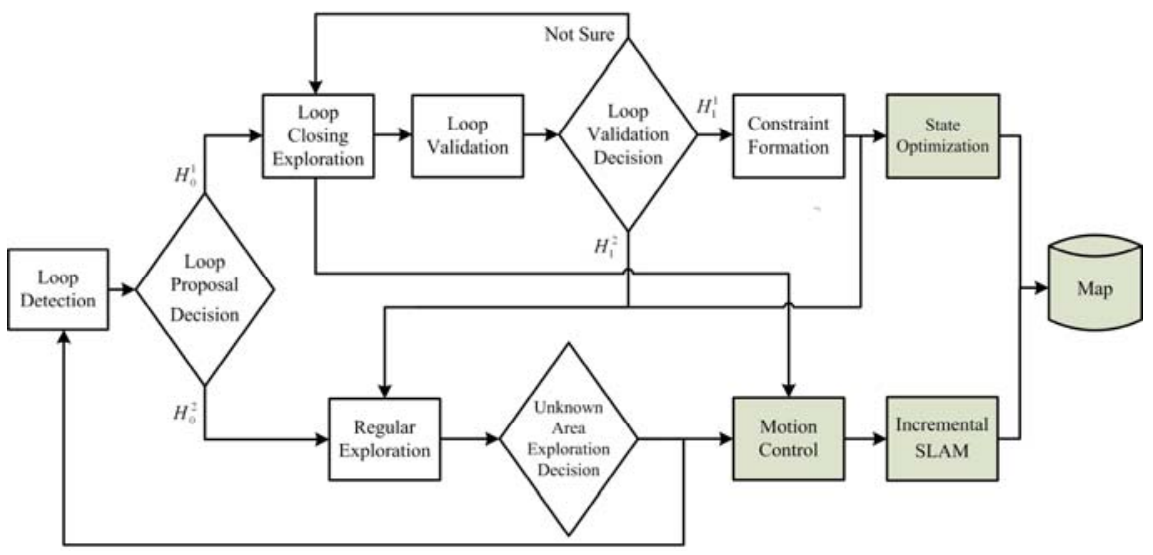

Fig. 1. The flow chart of our active loop closing process. The diamond frames are decision processes, and the shadowed frames are other processes not for loop closing. 
process will begin to execute. At every time step of the loop closing exploration, the loop validation decision process determine to accept the loop closure proposal, reject it, or be not sure about it. If it's not sure, the loop closing exploration continues. If the loop closure proposal is accepted, the loop constraint formation process will be activated, and then the robot reenters into the regular exploration mode. When loop closure proposal is rejected, the robot will reenter into the regular exploration mode directly.

\section{Active Loop Closing Decision Processes}

In this section, we will describe the detailed implements of every decision process in our active loop closing architecture.

\subsection{An Auxiliary Topological Map for Decisions}

In order to facilitate the decisions in our active closing process, we develop an auxiliary topological map $G$, which is composed by discrete points of robot's path and the entrances of unknown areas. The entrance of an unknown area is represented by the midpoint of the continuous border between explored free-space and the unexplored area. The entrance are called frontiers [8], and denoted by $f_{i}, i=1, \ldots, N$, where $N$ is the number of frontiers. The set of all frontiers is denoted as $F=\left\{\left.f_{i}\right|_{i=1, \ldots, N}\right\}$. The direction of a frontier is defined as the normal direction of the border from the explored area to the unexplored. To construct $G$, we initialize it with using the starting location of the robot. The present robot location $x_{t}$ will be added to $G$ if the distance between $x_{t}$ and all other path nodes of $G$ exceeds a certain threshold $c_{1}$, or if all other path nodes of $G$ are invisible to $x_{t}$ :

$$
G^{t}=\left\{x_{t} \cup G^{t-1}\right\} \text { if } \forall x_{i} \in G^{t-1}:\left[\operatorname{dist}\left(x_{t}, x_{i}\right)>c_{1} \vee \operatorname{invisible}\left(x_{t}, x_{i}\right)\right] .
$$

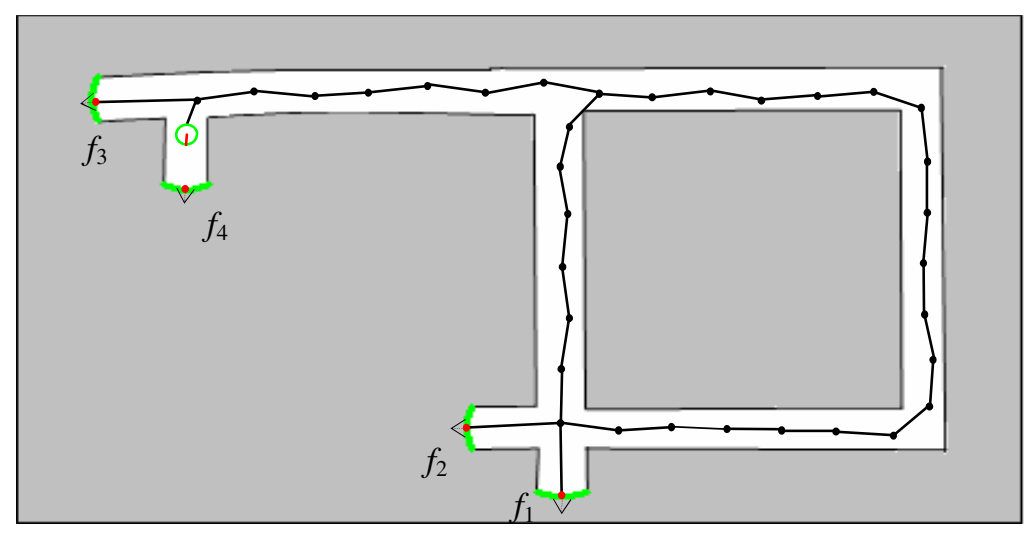

Fig. 2. A sketch of a topological map and frontiers. Three frontiers (green lines) are added to the topological map (black and red points and lines between them). Frontier $f_{4}$ is being explored, so deleted from the topological map. 
After $x_{t}$ is added, an edge between $x_{t}$ and the nearest and local reachable point $x_{i}$ is attached to $G$. Any frontier will be added to $G$ at the moment it was founded, at the same time an edge between the frontier and the nearest path node in $G$ which is visible to the frontier is also attached to $\mathrm{G}$.

Fig. 2 is a sketch map of the topological map and frontiers. In this figure, white area is explored free-space, and gray area is unknown. The black lines between them are obstacle borders. The black points are path nodes, and the lines between them are edges of the topological map. There are four frontiers $f_{1}, f_{2}, f_{3}, f_{4}$, indicated by green lines and red points. Because $f_{4}$ is being explored, it isn't added to the topological map.

\subsection{Loop Proposal Decision}

If the robot has returned to a previously explored area along a loop path, it must enter the area from a frontier in the topological map $G$. Supposing that the present robot position (location and facing) estimation is $\hat{x}_{t}$, and the error covariance matrix is $\Sigma_{t}$, which can be obtained by most incremental SLAM algorithms. Then $\left(x-\hat{x}_{t}\right)^{T} \Sigma_{t}^{-1}\left(x-\hat{x}_{t}\right)$ follows the $\chi_{d}^{2}$ distribution of dimension $d=\operatorname{dim} x$. The following set

$$
R_{x}=\left\{x \mid\left(x-\hat{x}_{t}\right)^{T} \Sigma_{t}^{-1}\left(x-\hat{x}_{t}\right)<\delta_{\sigma_{1}}\right\}
$$

is a confidential interval around $\hat{x}_{t}$ with the confidential level $1-\sigma_{1}$. We define a set of frontiers in $G$ as

$$
I=\left\{f_{i} \in G \mid \bar{f}_{i} \in R_{x}, \operatorname{dist}_{G}\left(f_{i}, \hat{x}_{t}\right)>c_{2}, i=1, \ldots, N\right\},
$$

where $\operatorname{dist}_{G}(x, y)$ is the distance between the node $x$ and $y$ in $G, c_{2}$ is a threshold insuring that $f_{i}$ is enough far away from $\hat{x}_{t}$, and $\bar{f}_{i}$ is the point with the same position and contrary direction with $f_{i}$. If $I \neq \varnothing$, there are some frontiers around $\hat{x}_{t}$, so the robot possibly has entered a previously explored area. Then the hypothesis $H_{0}^{1}$ is accepted, a potential loop is proposed, and the loop closing exploration is activated. Otherwise, the robot continues the regular exploration.

\subsection{Unknown Area Exploration Decision}

During the regular exploration, there may be many frontiers available for the robots to explore. The method to decide which one is to explore is called exploration strategy. Usual methods choose the nearest or biggest frontiers [8]. In our active loop closing approach, the exploration strategy is simple: trying to select the frontier most likely to close a loop, at the same time the exploration cost is also taken into account.

Let $C(i)$ denotes the exploration cost of frontier $f_{i}$, and $U(i)$ denotes its loop closing utility. Then our unknown area exploration decision can be expressed as

$$
i^{*}=\underset{i}{\arg \max }[U(i)-C(i)] .
$$


The cost and utility of each frontier can be calculated as follows.

Cost: The exploration cost of frontier $f_{i}$ is given by the minimum path from the robot location to the frontier in the map built by far:

$$
C(i)=\operatorname{dist}\left(\hat{x}_{t}, f_{i}\right) \text {. }
$$

The minimum path can be computed efficiently by graph searching methods such as the $A^{*}$ algorithm or Dijkstra's algorithm.

Utility: In our approach, the loop closing utility of each frontier is evaluated by the possibility to close a loop when the robot explore at that frontier. In our approach, the possibility of a frontier is evaluated by the distance and direction from it to others:

$$
U(i)=\max \left\{-k_{i j} \times \operatorname{mdist}\left(f_{i}, f_{j}\right) \mid \operatorname{dist}_{G}\left(f_{i}, f_{j}\right)>c_{3}, j=1,2, \ldots, N \text { and } i \neq j\right\},
$$

where, $\operatorname{mdist}\left(f_{i}, f_{j}\right)$ is the Mahalanobis distance of $f_{i}$ and $f_{j}$, which is calculated in a new coordinate system with the direction of $f_{i}$ as an axis. In order to avoid robots' influence to the local exploration, distance of $f_{i}$ and $f_{j}$ in the topological map $G$ must larger than a certain threshold $c_{3}$. $k_{i j}$ is a punishment coefficient for the directions of $f_{i}$ and $f_{j}$. If $f_{i}$ and $f_{j}$ are in the direction of being closer, then $k_{i j}=1$. Otherwise, $k_{i j}$ is calculated by the following formula:

$$
k_{i j}=\Delta \theta_{i j} / 90^{\circ} \text {, }
$$

where, $\Delta \theta_{i j}$ is the minimum angle about which the robot must turn around if it explores ahead through $f_{i}$ to close a loop at $f_{j}$. For example, in Fig. $2 k_{42}=1.0, k_{32} \approx 2.0$ and $k_{31} \approx 3.0$.

As shown in Fig. 2, according to the design of the exploration cost and utility, the unknown area exploration decision process selects the frontier $f_{4}$ to explore.

\subsection{Particle-Filter-Based Loop Validation Decision}

The task of the loop validation decision is to decide whether or not the robot has truly entered a previously explored area using sensor readings. The straightforward approach would be to compare the present local map with the previously built map. However, such an approach could be extremely inefficient since the searching space is too huge. An alternative approach is to localize the robot in the previously built map $[5,6]$. If the robot could localize itself well in the previously built map, we believe that the robot has entered into it, and make sure that there is a loop.

The localization problem for mobile robot comes in two flavors: global localization and position tracking. Since the initial robot position in the previously built map, which denoted as $M_{e}$, is not known, the loop validation is a global localization. Recently the Monte Carlo localization, also called Particle Filter, has been proven to be a practical, efficient and robust global localization approach and been used widely [9]. The loop validation through the global localization in $M_{e}$ is different from the classical global localization problem. Since at the beginning of the validation, the robot may be not in $M_{e}$, it's essential a global localization problem in a partial map and the classical particle filter is incompetent for the loop validation decision. 
We have modified the classical particle filter, and put forward a global localization algorithm in a particle map based on the work of Fox [11]. Similar to the implementation of classical particle filter, our algorithm represents the posterior distribution of the robot position at each time step by a set of particles with importance weights: $S_{t}=\left\{\left\langle x_{t}^{[i]}, w_{t}^{[i]}\right\rangle \mid i=1, \ldots, N_{t}\right\}$, where $N_{t}$ is the number of particles. The algorithm updates the particle set according to the robot movement and sensor readings.

Table 1 outlines the algorithm. Suppose that the loop validation starts at time step 0, and $N_{0}$ particles with uniform importance weights are initialized in $M_{e}$ following the distribution of the robot position estimation by far $p\left(x_{0}\right)$. We don't know when and where the robot enters into $M_{e}$, but it must enter $M_{e}$ through the frontier. At each iteration of the particle filter, a little of $\varepsilon N_{t}$ new particles starting nearby the frontier (Step 7) are generate following the distribution of the robot position estimation by far $p\left(x_{t}\right)$. Because there are new particles added at every time step, the algorithm resamples particles at every time step. In case of deleting the particles near the enter point of the robot in the resampling step, the algorithm will keep these new particles unresampled for a fixed time $\Delta t$ and just adjust their importance weight. So at every time step $t$ the particles can be divided into two parts: new particles generated in time interval $[t-\Delta t, t]$, which are call frontier particles, the set of them is denoted as $S_{t}^{f}$ and their number is $N_{t}^{f}$. Others are called inner particles, their set is denoted as $S_{t}^{i}$ and their number is $N_{t}^{i}$. So

$$
S_{t}=S_{t}^{f} \cup S_{t}^{i}
$$

At time step $t+1$, those frontier particles generated before $t-\Delta t$ in $S_{t+1}^{f}$ will become inner particles and are put into $S_{t+1}^{i}($ Step 5). The algorithm resamples the particles in $S_{t}^{i}$ (Step 11), and just adjusts the importance weights of the frontier particles (Step 9).

If a wrong loop validation decision is made, an inconsistent map will be generated, the robot may get lost in the inconsistent map and the exploration task will fail. So the loop validation decision is critical to exploration and mapping of the autonomous robot, and it must be greatly careful to make a decision. When there are no enough sensing evidences to support $H_{0}^{2}$ and $H_{1}^{2}$, the robot should to continue the loop closing exploration. Yet, the robot couldn't take the loop closing exploration ceaselessly, and there should be some means to end such a ceaseless exploration. Supposing the present robot position estimation at the beginning of the loop closing exploration is $\hat{x}_{0}$, and the estimation error covariance matrix is $\Sigma_{0}$, then the following set:

$$
P=\left\{x \mid\left(x-\hat{x}_{0}\right)^{T} \Sigma_{0}^{1}\left(x-\hat{x}_{0}\right) \leq \delta_{\sigma_{2}}\right\}
$$

is a confidential interval around $\hat{x}_{0}$ with the confidential level $1-\sigma_{2}\left(\sigma_{2}<\sigma_{1}\right)$. If at time $t^{\prime}$ the robot position estimation $\hat{x}_{t^{\prime}} \notin P$, then we believe that if there really is a loop, then the robot must have entered into $M_{e}$, no new frontier particles are generated any more, and the loop closing validation decision must be made in limited time. 
Table 1. Outline of the particle filter based localization algorithm for loop validation decision

1. Inputs: $S_{t-1}^{i}=\left\{\left\langle x_{t-1}^{[j]}, w_{t-1}^{[j]}\right\rangle \mid j=1, \ldots, N_{t-1}^{i}\right\}, S_{t-1}^{f}=\left\{\left\langle x_{t-1}^{[k]}, w_{t-1}^{[k]}, c n t_{t-1}^{[k]}\right\rangle \mid k=1, \ldots, N_{t-1}^{f}\right\}$, control information $u_{t}$,observations $z_{t}$, particle map $M_{e}$

2. $S_{t}^{i}=\varnothing, S_{t}^{f}=\varnothing ; \Omega_{t}^{i}=0 ; \Omega_{t}^{f}=0 ; \quad$ I/Initialize $N_{t}^{i}=N_{t-1}^{i} ; N_{t}^{f}=N_{t-1}^{f}$;

3. for all samples in $S_{t-1}^{i}$ do $x_{t}^{[j]} \sim p\left(x_{t} \mid x_{t-1}^{[j]}, u_{t}\right) ;$ //Generate $S_{t}^{i}$ $w_{t}^{[j]}=w_{t-1}^{[j]} p\left(z_{t} \mid x_{t}^{[i]}\right)$; //Predict $S_{t}^{i}:=S_{t}^{i} \cup\left\{x_{t}^{[j]}, w_{t}^{[j]}\right\}$; $\Omega_{t}^{i}=\Omega_{t}^{i}+w_{t}^{[j]} ;$ //Update importance weights //Compute the sum of importance weights

4. for all samples in $S_{t-1}^{f}$ do $x_{t}^{[k]} \sim p\left(x_{t} \mid x_{t-1}^{[k]}, u_{t}\right)$; // Generate $S_{t}^{f}$ $w_{t}^{[k]}=w_{t-1}^{[k]} p\left(z_{t} \mid x_{t}^{[i]}\right)$; //Predict cnt $_{t}^{[k]}++$; // Update importance weights //Count the time step of particle $k$

5. if $\left(c n t_{t}^{[k]}>\Delta t\right)$ then // Change frontier particles generated $S_{t}^{i}:=S_{t}^{i} \cup\left\{x_{t}^{[k]}, w_{t}^{[k]}\right\}$; // before $t-\Delta t$ into inner particles $N_{t}^{i}=N_{t}^{i}+1 ; \quad N_{t}^{f}=N_{t}^{f}-1 ; \quad$ // Update the size of $S_{t}^{i}$ and $S_{t}^{f}$ $\Omega_{t}^{i}=\Omega_{t}^{i}+w_{t}^{[k]} ;$

6. else

$$
\begin{aligned}
& S_{t}^{f}:=S_{t}^{f} \bigcup\left\{x_{t}^{[k]}, w_{t}^{[k]}, c n t_{t}^{[k]}\right\} ; \\
& \Omega_{t}^{f}=\Omega_{t}^{f}+w_{t}^{[k]} ;
\end{aligned}
$$

7. for $i=1: \varepsilon N_{t} \quad$ do // Generate new frontier particle $x_{t}^{[i]}=$ sample_near_frontiers $\left(M_{e}\right) ; \quad / /$ Sample near the frontier $w_{t}^{[i]}=p\left(z_{t} \mid x_{t}^{[i]}\right) / N_{t} ; c n t_{t}^{[i]}=1 ; \quad$ // Update importance weights $S_{t}^{f}:=S_{t}^{f} \cup\left\{x_{t}^{[i]}, w_{t}^{[i]}, c n t_{t}^{[i]}\right\}$; $\Omega_{t}^{f}=\Omega_{t}^{f}+w_{t}^{[k]}$;

8. $N_{t}^{f}=N_{t}^{f}+\varepsilon N_{t}$; //Compute the number of particles in $S_{t}^{f}$

9. for all samples in $S_{t}^{f}$ do //Normalize importance weights in $S_{t}^{f}$ $w_{t}^{[k]}=w_{t-1}^{[k]} /\left(\Omega_{t}^{i}+\Omega_{t}^{f}\right) ;$

10. $N_{t}^{i}=N_{t}-N_{t}^{f}$; // Update the size of $S_{t}^{i}$

11. $w_{t}^{i}=\Omega_{t}^{i} /\left[\left(\Omega_{t}^{i}+\Omega_{t}^{f}\right) N_{t}^{i}\right]$; //Compute importance weight of every inner

12. resample $\left(S_{t}^{i}, N_{t}^{i}, w_{t}^{i}\right)$; // particle I/Resample $S_{t}^{i}$ 
In the loop validate decision, all inner particles are used to test $H_{0}^{2}$ and $H_{1}^{2}$. The center of all the inner particles is

$$
\hat{x}_{t}=\frac{1}{N_{t}^{i}} \sum_{j=1}^{N_{t}^{i}} x_{t}^{[j]} w_{t}^{[j]} .
$$

$w_{t}^{[i]}$ is an importance weight before resampling. Let $Q$ is a set around $\bar{x}_{t}$ such that

$$
Q=\left\{x \mid:\left\|x-\bar{x}_{t}\right\|<\delta_{q}\right\},
$$

where $\delta_{q}$ is a threshold to control the volume of $Q$. We make the loop validation decision by using the mean of the importance weights of all the inner particles before resampling and the sum of them after resampling respectively:

$$
\bar{w}=\frac{1}{N_{Q}} \sum_{x_{t}^{[i]} \in Q} w_{t}^{\prime[i]}, \Omega_{q}=\sum_{x_{t}^{[i]} \in Q} w_{t}^{[i]},
$$

Where $N_{Q}$ is number of the inner particles in $Q$, and $w_{t}^{[i]}$ is an importance weight after resampling. When $\bar{w}$ and $\Omega_{q}$ are all big enough:

$$
\bar{w}>\eta_{1} \vee \Omega_{q}>\left(1-\sigma_{3}\right) N_{t}^{i} / N_{t},
$$

we will accept $H_{0}{ }^{2}$ and make sure that there really is a loop. After the time step $t^{\prime}+\Delta t_{1}$, if $H_{0}{ }^{2}$ is still not accepted and $\bar{w}$ and $\Omega_{q}$ are all small enough:

$$
\bar{w}<\eta_{2} \vee \Omega_{q}<\left(1-\sigma_{4}\right) N_{t}^{i} / N_{t},
$$

we will accept $H_{1}{ }^{2}$ and reject the loop proposal. Otherwise, we believe that there is no enough sensing evidence to make a decision and continue the loop closing exploration. After the time step $t^{\prime}+\Delta t_{2}\left(\Delta t_{2}>\Delta t_{1}\right)$, if neither $H_{0}{ }^{2}$ or $H_{1}{ }^{2}$ is accepted, we will accept $H_{1}{ }^{2}$ and return to regular exploration in case of a ceaseless loop closing exploration. How to set $\delta_{q}, \eta_{1}, \sigma_{3}, \eta_{2}, \sigma_{4}, \Delta t_{1}$ and $\Delta t_{2}$ is related to the perception models of sensors and the environments, and can be learned from experiments [9].

Once we have accepted $H_{0}^{2}$, we will simultaneously get a robot position estimation in $M_{e}$, and a loop closure constraint is established. We also can use the particle filter as a path smoother to localize the path of the robot $M_{e}$ and establish multiple constraints for more precise state optimization.

\section{Experiments}

We used a simulation environment just like the open experiment dataset: ut_austin_ aces (http://cres.usc.edu/radishrepository/outgoing/ut_austin_aces3/aces3_publicb.rtl) described to test our active loop closing method. Because we use a different exploration strategy which results in a different exploration path, the real experiment dataset couldn't be used. Our simulation environment is about $90 \mathrm{~m} \times 90 \mathrm{~m}$, has four adjoining loops, as shown in Fig.4.a, and the robot needs to run about 540m to explore of the whole environment. In the simulation experiment, a simulated laser range finder is 


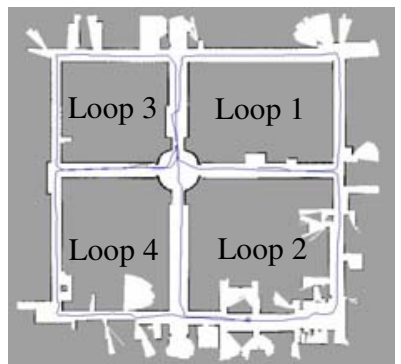

(a)

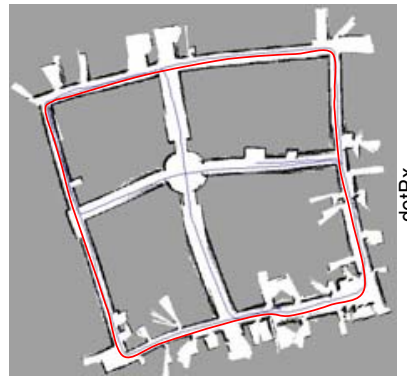

(d)

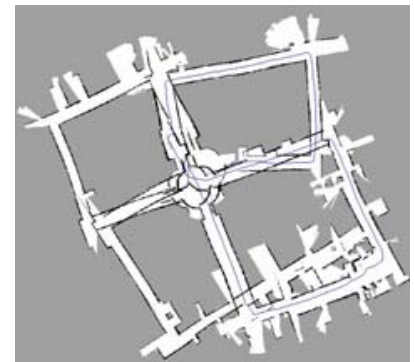

(b)

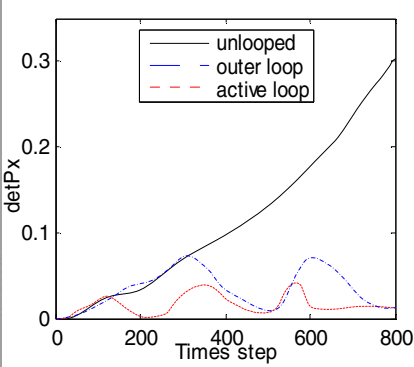

(e)

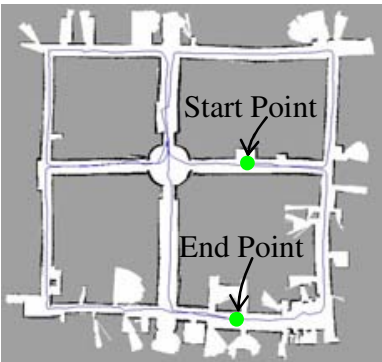

(c)

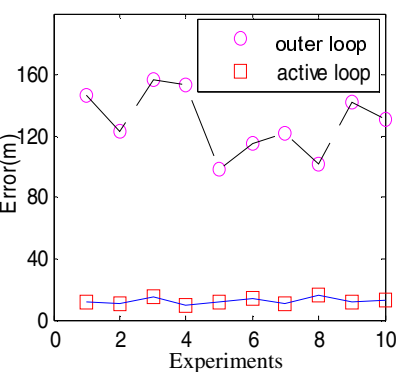

(f)

Fig. 3. Experiment result. (a) The map and robot path without error. (b) Incremental map and path. (c) The map and path after active loop closing. (d) The map and path when first explore the outer loop (read line). (e) The changing of the determinant of the robot position error covariance matrix with time. (f) The sums of the absolute robot position error of the whole path in ten experiments.

used, Histogram Correlation is used to incrementally build grid maps [11], and a local map is maintained and registered to a global map at intervals.

Fig. 3 shows our experiments results. Fig. 3(a) depicts the map and robot path (blue line) without error. According our active loop closing exploration strategy, the robot explores Loop 1 firstly, then Loop 2 and Loop 3, at last Loop 4 is explored. Fig. 3(b) is the incremental map and path without loop closure optimization. Obviously, the map is inconsistent, and can't be used for robot navigation. Fig. 3(c) is the map and path obtained through our active loop closing approach and optimized by the method put forward by Lu etc [4], and Fig. 3(d) is the optimized map and path when the robot explored the big outer loop (red line) first and then the middle part. They show that the map obtained by our active loop closing approach is much more precise. Fig. 3(e) compares the changing of the determinant of the robot position error covariance matrix with time in deferent situations: incremental mapping, our active loop closing and firstly exploring the outer loop. And Fig. 3 (f) depicts the sums of the absolute robot position error of the whole path in ten experiments by two different exploration strategies: our active loop closing and firstly exploring the outer loop. It shows that the average sum of the absolute robot position error of the later strategy is about 10 times larger than our approach. So our active loop closing approach can improve the mapping precision greatly and efficiently.

In the loop proposal decision, we set $\sigma_{1}=0.05$ to ensure most potential loops can be proposed. In the implementation of the particle filter for the loop validation decision, 


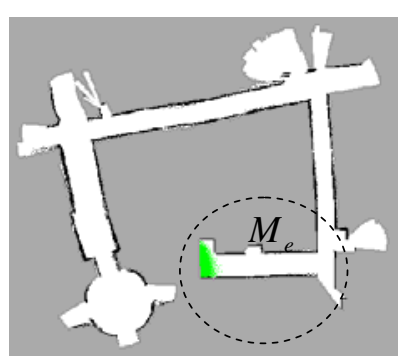

(a)

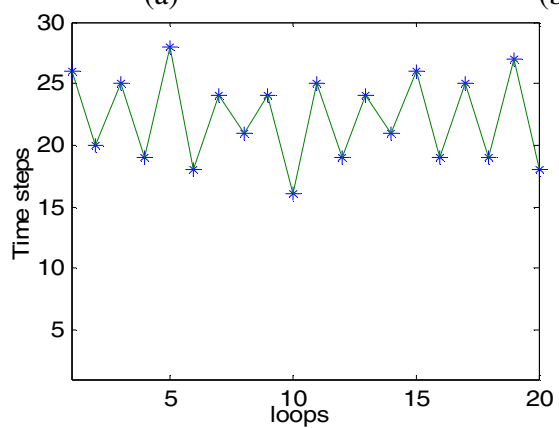

(d)

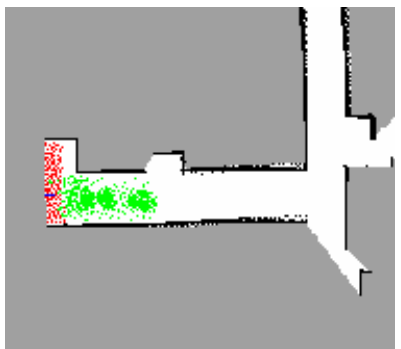

(b)

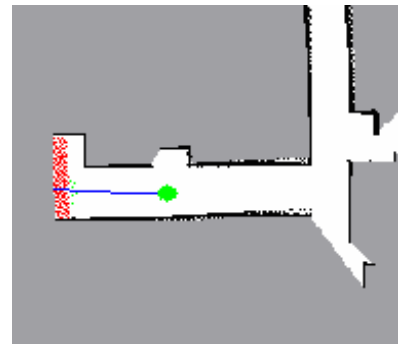

(c)

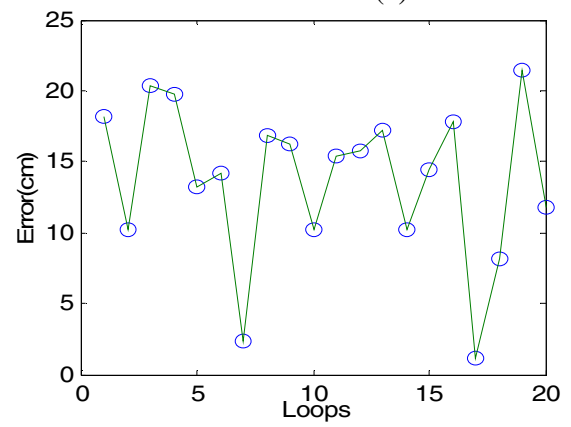

(e)

Fig. 4. Experiment results of particle-filter-based loop validation. (a) Particles (green points) at the initial time step. (b) Particles (red points are frontier particles and green ones are inner particles) when the robot enter $M_{e}$. (c) Particles when loop closure was validated. (d) Time steps needed to converge for 20 loops. (e) Position error at the converged points for 20 loops.

1200 particles are used and each iteration step takes less than 0.1 s on a notebook PC with $1.73 \mathrm{CPU}$ and $512 \mathrm{Mb}$ memories, so the algorithm can be used in real time. The width of a frontier is set as $0.5 \mathrm{~m}$. The ratio of new frontier particles is set as $\varepsilon=8 \%$, and they are kept for two time steps to be not resampled. So there are at most 192 frontier particles. We have found that when $\varepsilon$ is more than $4 \%$ and less than $12 \%$, the algorithm all could validate an existing loop successfully. When the particles near the center of inner particles is more than $90 \%$, the loop validation will accept $H_{0}{ }^{2}$ to believe that for a certainty a loop is closed. Fig. 4(a) shows the initiation of particles following the distribution of robot position estimation at the beginning of the loop validation. Fig. 4(b) shows the particles at the time when the robot entered into $M_{e}$, the red points are frontier particles and the green ones are inner particles. Fig. 4(c) depicts distribution of particles at the time when $H_{0}{ }^{2}$ was accepted.

Because the range of $M_{e}$ is not too big and we have a prior distribution of the robot localization, especially have a prior knowledge about the facing of the robots, so the particle filter can converge very fast once the robot enter into $M_{e}$. Fig. 4(d) shows the time step at which a loop is validated in 20 loop closing processes of 5 experiments. Fig. 4(e) depicts the absolute localization error of at the point where loops were validated, and the error is some large, and this is the common faults of particle-filterbased localization approaches. In order to improve the precision of state optimization, we have saved all the sensor readings during the loop validation process, and used the particle filter as a path smoother to gain multiple constraints. 


\section{Conclusion}

The loop closing is an open problem of SLAM. In this paper, a decision-theoretic active loop closing approach is presented, which integrate exploration strategy with loop closing process intending to find and close loops as soon as possible to reduce the mapping error. The active loop closing process is modeled as a multi-stage decision problem, and a frontier-based auxiliary topological map is introduced to facilitate decision making. Our approach is a macro exploration strategy, and doesn't conflict with usual exploration strategy [12], so has less effect on local exploration. In addition, a particle-filterbased localization method used in partial map is put forward for loop validation, which can validate loop closure and build loop constraints correctly and efficiently. Experiments has shown that our active loop closing approach can detect and close loops successfully in real time, and at the same time improve the mapping precision efficiently.

Our active loop closing approach is essential a loop closing architecture, and doesn't limited to any particular incremental SLAM method. In the future work, we will try to use this method in vision-based SLAM for the RoboCup Rescue Competition.

\section{References}

1. Dissanayake, M.W., Newman, P., Clark, S., Durrant-Whyte, H.F., Csorba, M.: A Solution to the Simultaneous Localization and Map Building (SLAM) Problem. IEEE Transactions on Robotics and Automation 17, 229-241 (2001)

2. Andrea, C., Gian, D.T.: Lazy Localization Using a Frozen-Time Filter, http://purl.org/censi/research/2008-icra-fts.pdf

3. Ji, X.-C., Zheng, Z.-Q., Hui, Z.: Robot Position Convergency in Simultaneous Localizaton and Mapping. In: IEEE Intl. Conf. on Mechatronics and Automation, pp. 320-325. IEEE, Harbin (2007)

4. Lu, F., Milios, E.: Globally Consistent Range Scan Alignment for Environment Mapping. Autonomous Robots 4, 333-349 (1997)

5. Gutmann, J.S., Konolige, K.: Incremental Mapping of Large Cyclic Environments. In: Proceedings of the IEEE International Symposium on Computational Intelligence in Robotics and Automation, Monterey, CA, USA, pp. 318-325 (1999)

6. Neira, J., Tardos, J.D., Castellanos, J.A.: Linear Time Vehicle Relocation in SLAM. In: IEEE Intl. Conf. on Robotics and Automation, Taipei, Taiwan, pp. 427-433 (2003)

7. Stachniss, C., Hahnel, D., Burgard, W., Grisetti, G.: On Actively Closing Loops in GridBased FastSLAM. Advanced Robotics 19, 1059-1079 (2005)

8. Yamauchi, B.: Frontier-Based Exploration Using Multiple Robots. In: Proceedings of the 2nd International Conference on Autonomous Agents, pp. 47-53. ACM, New York (1998)

9. Thrun, S., Fox, D., Burgard, W., Dellaert, F.: Robust Monte Carlo Localization for Mobile Robots. Artificial Intelligence 128, 99-141 (2001)

10. Fox, D.: Distributed Multi-Robot Exploration and Mapping. In: Proceedings of the 2nd Canadian Conference on Computer and Robot Vision, pp. 15-25. IEEE Press, Los Alamitos (2005)

11. Thomas, R.: Using Histogram Correlation to Create Consistent Laser Scan Maps. In: IEEE Intl. Conf. on Robotics Systems, pp. 625-630. IEEE, EPFL, Lausanne (2002)

12. Gonzalez-Banos, H.H., Latombe, J.: Navigation Strategies for Exploring Indoor Environments. International Journal of Robotics Research 21, 829-848 (2002) 